\title{
EVALUATION OF MULTIELECTRON IONIZATION CROSS-SECTIONS IN ENERGETIC ION-ATOM COLLISIONS
}

\author{
Igor D. Kaganovich, Edward Startsev and Ronald C. Davidson, \\ Plasma Physics Laboratory, Princeton University, Princeton, NJ 08543, USA
}

\begin{abstract}
The use of heavy ions beams with mass of order 100 atomic mass units and energy of order $10 \mathrm{MeV}$ per nucleon has been proposed as a driver for heavy ion fusion. Multi-electron-loss collisions of the beam ions with the background gas atoms result in an increase in the ion charge, which is an unwanted effect for beam propagation through the accelerator and transport regions, and the target chamber. Knowledge of the electron-loss cross-sections is essential to understand and address this issue. This paper describes the theoretical methods employed for calculation of the cross-sections.
\end{abstract}

\section{INTRODUCTION}

To describe the collision cross-sections of heavy particles it is natural to use the eikonal approximation as described in Ref.1. In this approximation, the scattering amplitude for inelastic transitions from initial state $i$ to final state $f$ is given by [1]

$$
f_{i f}=\frac{k^{\prime}}{2 \pi i} \int<i|S| f>e^{i q \rho} d^{2} \rho,
$$

where $\boldsymbol{p}$ and $\boldsymbol{p}^{\prime}$ are the particle momenta before and after the collision, $k^{\prime}=p^{\prime} / \hbar, \hbar \boldsymbol{q}=\boldsymbol{p}^{\prime}-\boldsymbol{p}, \rho$ is the impact parameter, and

$$
\begin{aligned}
& S\left(\rho, r_{P}, r_{T}\right)=\exp \left(-i \Psi\left(\rho, r_{P}, r_{T}\right)\right), \\
& \Psi\left(\rho, r_{P}, r_{T}\right)=\frac{1}{\hbar V} \int_{-\infty}^{\infty} U\left(\rho, r_{P}, r_{T}, z\right) d z,
\end{aligned}
$$

where $U(r)$ is the sum of interaction potentials between all projectile electrons (at positions $\boldsymbol{r}_{\boldsymbol{P}}$ ) and target electrons and nuclei (at positions $\boldsymbol{r}_{T}$ ), and $V$ is the relative velocity. Conservation of energy requires that [1]

$$
(\hbar \boldsymbol{q})^{2}=\left(\frac{E_{f}-E_{i}}{V}\right)^{2}+(M V \theta)^{2},
$$

where $E_{i}$ and $E_{f}$ are the initial and final energy states, $\mathrm{M}$ is the mass of the projectile, and $\theta$ is the scattering angle. The total cross-section $\sigma_{i f}$ is the integral of the square of the scattering amplitude over the scattering angle. Making use of Eq.(4), $\sigma_{i f}$ can be expressed in the form [1]

$$
\sigma_{i f}=\int d^{2} q \frac{\left|f_{i f}\right|^{2}}{k^{2}}
$$

The total cross-section for electron loss from the projectile particle is determined by integrating over all possible final states of the target atom and energies of the ionized electrons in Eq.(5).

\section{APPROXIMATIONS EMPLOYED FOR CROSS-SECTION CALCUALTIONS}

The calculation of $\sigma_{i f}$ by using Eq.(5) is straightforward but rather cumbersome, which is why a number of approximations are desirable. If $\Psi(\rho)<<1$ for all $\rho$, then $\exp (-i \Psi(\rho)) \approx 1-i \Psi(\rho)$, and Eq.(2) coincides with the Born-approximation. For neutral target atoms with nucleus charge $Z_{T}, \Psi(\rho)$ has a maximum at $\rho=0$, where $\Psi(0) \sim Z_{T} e^{2} /(\hbar V)$, and the condition for validity of the Born-approximation for all impact parameters can be expressed as

$$
\frac{Z_{T} e^{2}}{\hbar V}<<1
$$

In the opposite limit to Eq.(6), the situation is more complicated. Because $\Psi(\rho)$ is a rapidly decreasing function of impact parameter $\rho$, one can separate regions where $\Psi(\rho)<1$ for $\rho>\rho^{*}\left[\Psi\left(\rho^{*}\right)=1\right]$ from the region where $\rho<\rho^{*}$ and $\Psi(\rho)>1$. In the region where $\rho>\rho^{*}$ the Born-approximation can be used. In the region where $\rho<\rho^{*}$, the quasi-classical approximation can be utilized. For example, this approach has been successfully used for the calculation of vibrational and rotational transitions in molecules [2]. Furthermore, if the momentum $p_{e}$ of the ionized electron is large enough that the uncertainty of the electron position is smaller than the target and projectile atomic radii,

$$
\Delta a=\hbar / p_{e}<<a_{T}, a_{P},
$$

a classical calculation can be used. Indeed, let us consider an ionizing collision under conditions opposite to the limit in Eq.(6). A high-energy ionized electron with momentum $p_{e}$, satisfying the inequality in Eq.(7), can be approximately described by the plane wave $\psi_{f} \sim \exp \left(i_{e} r / \hbar\right)$. Because the phases of the scattering amplitude in Eq.(2) and the electron wave function are large, the method of stationary phase can be applied for estimation of the integral $\langle i|S| f\rangle$. Thus, the main contribution to the integral is for

$$
\boldsymbol{p}_{e}=-\frac{1}{V} \int_{-\infty}^{\infty} \frac{\partial}{\partial \boldsymbol{r}_{P}} U\left(\rho, \boldsymbol{r}_{P}, z\right) d z,
$$

which corresponds to the classical calculation of electron scattering. 


\subsection{Born-Approximation}

If the inequality in Eq.(6) is satisfied, the Bornapproximation is valid for all impact parameters. It is convenient to recast the integral in Eq.(1) using Fourier transforms. Some straightforward algebra readily yields [3]

$$
\begin{aligned}
& \sigma_{i o n}=\frac{8 \pi e^{4}}{V^{2} \hbar^{2}} \int_{0}^{\infty} \sum_{l} d \varepsilon \int_{q_{\min }}^{\infty} \frac{d q}{q^{3}} P_{P}^{i o n}(\varepsilon, q, l)\left[Z_{T}^{i_{f T}}(q)\right]^{2} \\
& P_{P}^{i o n}(\varepsilon, q, l)=\left\langle\psi_{P}^{0}|\exp (i \vec{q} \vec{r})| \psi_{P}^{l}(\varepsilon)>\left.\right|^{2},\right. \\
& Z_{T}^{i_{f T}}(q)=Z_{T} \delta_{0 i_{f T}}-\sum_{j} F_{T}^{i_{f T}}(q)_{j}, \\
& F_{T}^{i_{f T}}(q)_{j}=<\psi_{T}^{0}\left|\exp \left(i \vec{q} \vec{r}_{j}\right)\right| \psi_{T}^{i_{f T}}>,
\end{aligned}
$$

where $q_{\min }$ is given by Eq.(4) with $\theta=0, P_{P}^{i o n}(\varepsilon, q, l)$ is the ionization transition strength of the projectile electron with final energy $\varepsilon$ and orbital momentum $l, i_{f T}$ represents the summation over all final states of the target atom, $\delta_{0 i_{f T}}$ is Kroniker function, $F_{T}^{i_{f T}}(q)_{j}$ is the inelastic form factor for the $j$ 'th electron of the target atom. Information on the inelastic atomic form factor (the final state is not the ground state) is rare and has to be calculated numerically. The ionization transition strength (10) has been calculated analytically only for one-electron ions [4]. For multi-electron ions, $P_{P}^{\text {ion }}(\varepsilon, q, l)$ also has to be calculated numerically.

Considerable simplification is possible if the difference in $q_{\min }$ for different final states can be neglected, approximating $q_{\min }=I / \hbar V$, where $I$ is the ionization potential. Thus, we obtain

$$
\begin{gathered}
\sigma_{i o n}=\frac{8 \pi e^{4}}{V^{2} \hbar^{2}} \int_{q_{\min }}^{\infty} \frac{d q}{q^{3}} P_{P}^{i o n}(q)\left[Z_{T}^{\text {eff }}(q)\right]^{2}, \\
P_{P}^{i o n}(q)=\int_{0}^{\infty} d \varepsilon \sum_{l} P_{P}^{i o n}(\varepsilon, q, l), \\
{\left[Z_{T}^{\text {eff }}(q)\right]^{2}=\sum_{i_{j T}}\left|Z_{T}^{i_{f T}}(q)\right|^{2} .}
\end{gathered}
$$

Here, $P_{P}^{\text {ion }}(q)$ is the probability of ionization of an electron with recoil momentum $q$ after a collision, and $Z_{T}^{\text {eff }}(q)$ is the effective charge of the target atom.

The summation in Eq.(14) is traditionally represented as a sum of the coherent (elastic, $i_{f T}=0$ ) and the incoherent scattering functions $\left(i_{f T} \neq 0\right)$. Numerical values of the coherent and incoherent scattering functions can be found in Ref. 8. Making use of the orthogonality and completeness of the wave functions, the incoherent scattering functions can be expressed in terms of the initial state [1]. Moreover, making use of Hartree's approximation for the atomic electron wave functions (neglecting Fock's exchange terms), we obtain the incoherent scattering function as a sum over individual electron form factors of the target atom [5]. This gives

$$
\begin{gathered}
{\left[Z_{T}^{e f f}(q)\right]^{2}=S_{T}^{e l}(q)+S_{T}^{\text {inc }}(q),} \\
S_{T}^{e l}(q)=\left(Z_{T}-F_{T}^{0}(q)\right)^{2}, F_{T}^{0}(q)=\sum_{j} F_{T}^{0}(q)_{j},
\end{gathered}
$$

$$
S_{T}^{i n c}(q)=N_{T}-\sum_{j}\left|F_{T}^{0}(q)_{j}\right|^{2},
$$

where $N_{T}$ is the number of electrons. Convenient analytical representations for the individual electron atomic form factors $F_{T}^{0}(q)_{j}$ are available for many atoms, e.g., see Refs. 6,7 and 8. For heavy atoms with very large $Z_{T}$, the Thomas-Fermi-Dirac model can be utilized [5].

Note that in the limit $q \rightarrow \infty$ and $F_{T}^{0}(q)_{j} \rightarrow 0$, the coherent $\quad\left(S_{T}^{e l} \rightarrow Z_{T}^{2}\right) \quad$ and incoherent scattering $\left(S_{T}^{\text {inc }} \rightarrow N_{T}\right.$ ) functions describe the interaction with the bare nucleus and with free atom electrons, respectively. In the opposite limit, $q \rightarrow 0$ and $F_{T}^{0}(q)_{j} \rightarrow 1$, the scattering functions for neutral atoms $\left(N_{T}=Z_{T}\right)$ tends to zero. Correspondingly, the function $\left[Z_{T}^{\text {eff }}(q)\right]^{2} / q^{3}$ for the neutral atoms tends to zero for large and small $q$ values, and therefore the values $q a_{T} \sim 1$ contribute to the integral in Eq.(9). Examples of good agreement between theory and experimental data can be found in Ref. 9.

Rough estimates can be made if $P_{P}^{\text {ion }}(q)$ is approximated by a step-function, i.e., zero for $m(\hbar q)^{2} / 2<I$, and unity otherwise. For large $I, q$ is also large and $Z_{T}^{\text {eff }}(q)^{2} \approx Z_{T}^{2}+Z_{T}$. In this case, Eq.(13) gives

$$
\sigma_{\text {ion }}=\frac{2 \pi e^{4} m}{V^{2}} \frac{Z_{T}^{2}+Z_{T}}{I} \text {. }
$$

\subsection{Impulse Approximation}

If the inequality in Eq.(6) is not satisfied, the Born approximation cannot be used. Nevertheless, separating large impact parameters with $\Psi(\rho)<<1$, the Born approximation can be used exclusively for $\rho>\rho *$. For impact parameters $\rho<\rho^{*}$ we can use the impulse approach [1] of instant interaction. The cross-section (13) derived in the Born-approximation is the product (integrated over all $q$ ), of the total differential crosssection of elastic and inelastic electron scattering with recoil momentum $q$, multiplied by the probability of ionization after the electron receives the recoil momentum $q$.

For the case opposite to the inequality in Eq.(6), the cross-section for electron scattering can not be calculated by use of the Born-approximation. Instead, we can use a classical calculation of the cross-section $d \sigma_{c l}(q)$. This gives

$$
\sigma_{\text {ion }}=\int_{q_{\text {min }}}^{\infty} P_{P}^{\text {ion }}(q) d \sigma_{c l}\left(q, \rho<\rho^{*}\right)+\sigma_{\text {ion }}{ }^{B}\left(\rho>\rho^{*}\right),
$$

where $d \sigma_{c l}(q)$ is calculated by accounting only for small impact parameters, $\rho<\rho^{*}$, and $\sigma_{i o n}{ }^{B}\left(\rho>\rho^{*}\right)$ is calculated from Eq.(13) accounting for only large impact parameters, $\rho>\rho^{*}$. In contrast to the classical trajectory 
method [10], Eq.(20) also incorporates classically forbidden transitions, both in the factor $P_{P}^{\text {ion }}(q)$, and particularly in $\sigma_{i o n}{ }^{B}\left(\rho>\rho^{*}\right)$. For example, in collisions with target ions, the long-range interactions for distances larger than the atomic radius, the ionization transitions are classically impossible (at large velocities the momentum transfer in Eq.(8) is too small to ionize). However quantum-mechanical effects with $\sigma_{i o n}{ }^{B}\left(\rho>\rho^{*}\right)$ contribute significantly to the cross-section.

\subsection{Comparison of the Classical and Born Approximations}

To illustrate differences between the classical and quantum-mechanical calculations, we have simulated stripping cross-sections for $\mathrm{He}^{+}$on $\mathrm{He}, \mathrm{N}$ and $\mathrm{Ar}$ atoms. The Born approximation calculations were carried out using Eq.(13). We compared the results with the Bohr formula, which is derived by calculating the classical scattering in the limit in Eq.(6). Under the conditions in Eq.(6), the distance of closest approach in a collision is much smaller than the atomic radius. Therefore, the collision can be viewed as a collision with a bare nucleus and free electrons. Applying the Rutherford formula readily yields

$$
\sigma^{\text {Bohr }}=\frac{2 \pi e^{4} m}{V^{2}} \frac{Z_{T}^{2}+Z_{T}}{I} .
$$

Interestingly, the Bohr formula (21), assuming classical scattering, coincides with the quantum-mechanical calculation in Eq.(19). This is a consequence of the fact that the Coulomb scattering is the same in quantummechanical and classical calculations [1]. However, under the condition in Eq.(6), the classical calculations are inaccurate because distance of closest approach is smaller than the electron wavelength [11]. In the general case, the Born-approximation results in Eq.(13) differ from the Bohr formula in Eq.(20). The Bohr formula does not account for screening of the nucleus charge, and consequently overestimates the cross-section when the parameter $Z_{T} e^{2} / \hbar V$ tends to unity. This can be corrected by calculating the electron scattering on the selfconsistent (average) atomic potential in Eq.(8). The contribution of electron density fluctuations, accounted for by the incoherent scattering function, decreases proportional to $1 / Z_{T}$.

As can be seen from Fig.1, the Bohr formula overestimates the cross-sections by at least $50 \%$, even in the best case of $\mathrm{He}^{+}-\mathrm{He}$ collisions. Classical calculations give results closer to the Born approximation crosssection than the Bohr formula in Eq.(21). For other cases, the difference between the classical calculation and the Born approximation may be larger. For example, calculation for a $20 \mathrm{MeV} / \mathrm{amu} \mathrm{N}^{+6}$ ion colliding with $\mathrm{He}$ gives a factor 2.7, and a factor of 1.8 for $\mathrm{N}$, and a factor of 2.0 for $\mathrm{Ar}$, for the difference between the classical and the Born approximations.

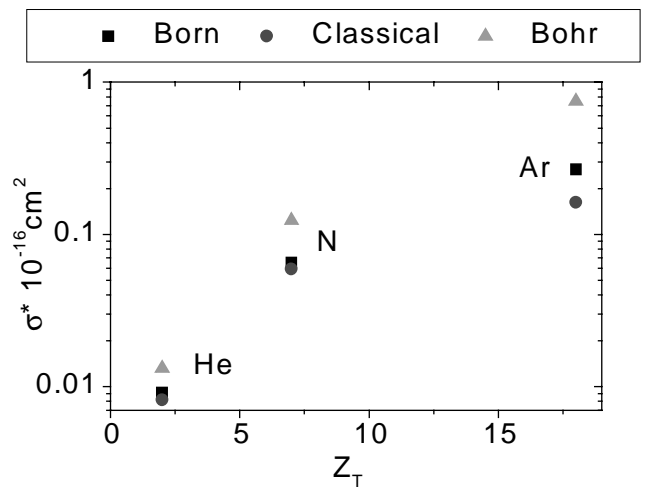

Fig. 1 Stripping cross-sections of $20 \mathrm{MeV} / \mathrm{amu} \mathrm{He}^{+}$ions colliding with $\mathrm{He}, \mathrm{N}$ and $\mathrm{Ar}$ atoms, calculated according to the Born approximation in Eq.(13), the Bohr formula in Eq.(20), and the classical estimate.

\section{CONCLUSIONS}

We have developed a robust method of calculating ionizing collisions for fast-moving ions through background atoms. The method use a combination of the Born approximation and the impulse approach. Typically, the Born approximation overestimates the cross-section, and the classical calculation underestimates the crosssection. The proposed approach eliminates both of these shortcomings.

Acknowledgment: This research was supported by the U.S. Department of Energy. It is a pleasure to acknowledge the benefit of stimulating discussions with S. Armel, L. Grisham, D. Mueller, G. Shvets, and V. Shevelko.

\section{REFERENCES}

[1] L.D. Landau and E.M. Lifshitz, Quantum Mechanics (Addison-Wesley Publishing Co., 1958).

[2] Yu.E. Gorbachev, I.D. Kaganovich, V.M. Strelchenya, Sov. Phys.- J.E.T.P. 68, 908 (1989).

[3] E. Merzbacher and H.W. Lewis, in Corpuscles and Radiation, Vol. 34 of "Encyclopedia of Physics", (ed. F. Flugge, Springer, Berlin, 1958).

[4] H. Bethe, Ann. Phys. (Leipz.) 5, 325 (1930).

[5] H. J. Hubbell, Wm. J. Veigele, E.A. Briggs et. al. J. Phys. Chem. Ref. Data 4, 471 (1975)

[6] G.H. Gillespie, Phys. Rev. A 18, 1967 (1978).

[7] Y.F. Chen, C.M. Kwei, and C.J. Tung, J. Phys. B:At. Mol. Opt. 261071 (1993)

[8] V.P. Shevelko, I. Yu. Tolstikhina and Th. Stoehlker, submitted to Nucl. Instr. Meth. (2001)

[9] D. Mueller, L. Grisham, I. Kaganovich, et.al., Physics of Plasmas, 8, 1753 (2001).

[10] R.E. Olson, J. Ulrich, and H. Schmidt-Bocking, Phys. Rev. A 39, 5572 (1989)

[11] N. Bohr, K. Dan. Vidensk. Selsk. Mat.- Fys. Medd. 18, N8 (1948). 